\section{Al-12 METABOLIC COMPETITION IN THE MICROENVIRONMENT IS A DRIVER OF CANCER PROGRESSION: A LESSON FOR LUPUS}

${ }^{1,4}$ Chih-Hao (Lucas) Chang ${ }^{*},{ }^{2,4}$ Jing Qiu, ${ }^{3,4}{ }^{2}$ rika Pearce. ${ }^{1}$ The Jackson Laboratory, Bar Harbor, ME, USA; ${ }^{2}$ Pfizer inc., San Diego, CA, USA; ${ }^{3}$ Department of Immunometabolism, Max Plunk Institute of Immunobiology and Epigenetics, Freiburg, Germany; ${ }^{4}$ Department of Pathology and Immunology, Washington University School of Medicine, St. Louis, MO, USA

\subsection{6/lupus-2018-Ism.12}

Background Properly functioning T-cells are critical for effective immunity. Upon activation, T-cells engage glycolysis and this metabolic adaptation is required for the optimal production of effector cytokines that mediate tumor clearance. However, during cancer T-cells often experience a progressive decline in effector functions, preventing tumor regression. Failure of T-cells to protect against cancer is thought to result from lack of antigen recognition, chronic activation, and/or suppression by other cells. Whether other mechanisms exist, or precisely how T-cell hyporesponsiveness in tumors is established, remains unclear.

Methods We used an established mouse sarcoma model of regressing and progressing tumors to compare the metabolic parameters between T-cells and tumor cells. By geneticallyengineering the glucose metabolism of regressing sarcoma cells, we were able to compare between groups based on tumor growth at the same background of antigenicity. Checkpoint blockade therapy (e.g. anti-PD-1, and anti-PD-L1 antibodies) were used to determine if the treatment can correct nutrient restriction experienced by $\mathrm{T}$ cells in a progressing tumor.

Results Glucose consumption by tumors metabolically restricts T-cells in the tumor microenvironment, which dampens their mTOR activity and glycolytic capacity, limits their interferongamma (IFN- $\gamma$ ) production, and leads to tumor progression. Enhancing glycolysis in an antigenic 'regressor' tumor is sufficient to override the ability of T-cells to respond to a major tumor rejection antigen, allowing progression of tumors that are normally rejected. Checkpoint blockade increases the metabolic fitness of T-cells and restore glucose in the microenvironment of progressing tumors, permitting T-cell glycolysis and IFN- $\gamma$ production.

Conclusions Metabolic competition in the tumor microenvironment dictates effector T-cell function and this influences cancer progression. Combining therapies that blunt tumor metabolism with those that promote glycolysis in $\mathrm{T}$ cells could provide new effective treatments for cancer. In lupus, research looking into metabolic requirement for immune cells and other modulators may provide novel insight to the nature of lupus development.

\section{Al-13 THERAPEUTIC INHIBITORS OF ANTIGEN PRESENTATION PATHWAYS IN SLE}

Maurice T Raycroft, Renelle Gee, Janie Merkel, Mark Mamula*. Yale University School of Medicine

10.1136/lupus-2018-Ism.13
Background The Mamula laboratory has identified and characterized a novel pathway of antigen presentation in autoimmune disease. In particular, B cells that acquire specific autoantigens can play an important role in triggering autoimmunity in both a direct and indirect manner. First, B cells can directly process autoantigen in triggering $\mathrm{T}$ cell autoimmunity. Therapeutics such as Rituxan and Belimumab have been developed to interfere with these direct functions of B cells. In the indirect pathway, autoantigen-bearing B cells form high affinity interactions with scavenger receptor A (SR-A), which is expressed primarily on macrophages (MФs) and dendritic cells (DCs). After receiving antigen from B cells, MФs and DCs are then highly competent in activating autoimmune $\mathrm{T}$ cells and initiating pathology in models of disease. Our working hypothesis is that identifying small molecules that inhibit the latter pathway will provide leads for developing a new treatment for autoimmunity.

Methods and results In brief, we have utilized probes to follow antigen presenting pathways between human B cells and MФs or DCs. As illustrated in the figure 1, B cells make direct contact with DCs and transfer antigen via a SR-A mediated pathway. We have also identified small molecule inhibitors (SMIs) that interfere with SR-A interactions and interrupt the transfer of autoantigen between B cells and DCs. MRL mice were treated with SMIs of SR-A and the onset and progression of autoimmunity (autoantibodies and autoreactive $\mathrm{T}$ cells) were assessed over time. SMI treatment of MRL mice greatly reduced autoAbs and $\mathrm{T}$ cell activation as well as tissue pathology.

Conclusions This study identifies a novel SR-A dependent pathway of antigen presentation between human B cells and MФs and DCs. Inhibition of this pathway reduces $\mathrm{T}$ cell activation, reduces the production of autoantibodies, and ameliorates kidney and skin pathology of SLE in a murine model.

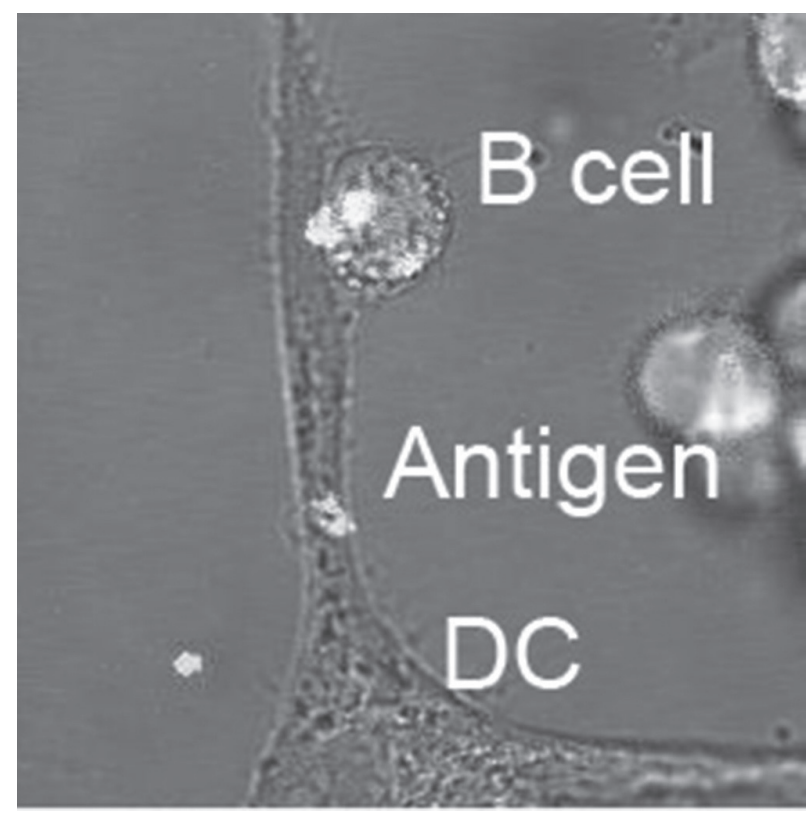

Abstract Al-13 Figure 1 